\title{
Syntactic Ambiguity Resolution Using A Discrimination and Robustness Oriented Adaptive Learning Algorithm
}

\author{
Tung-Hui Chiang, Yi-Chung Lin and Keh-Yih Su \\ Department of Electrical Engincering \\ National Tsing Hua University \\ Hsinchu, Taiwan 300, R.O.C. \\ E-Mail: thchiang@ee.nthu.edu.tw
}

\section{Topic Area: Computational Methods (Statistical), Application (NLP)}

\begin{abstract}
In this paper, a discrimination and robustness oriented adaptive learning procedure is proposed to deal with the task of syntactic ambiguity resolution. Owing to the problem of insufficient training data and approximation error introduced by the language model, traditional statistical approaches, which resolve ambiguities by indirectly and implicitly using maximum likelihood method, fail to achieve high performance in real applications. The proposed method remedies these problems by adjusting the parameters to maximize the accuracy rate directly. To make the proposed algorithm robust, the possible variations between the training corpus and the real tasks are also taken into consideration by enlarging the separation margin between the correct candidate and its competing members. Significant improvement has been observed in the test. The accuracy rate of syntactic disambiguation is raised from $46.0 \%$ to $60.62 \%$ by using this novel approach.
\end{abstract}

\section{Introduction}

Ambiguity resolution has long been the focus in natural language processing. Many rule-based approaches have been proposed in the past. However, when applying such approaches to large scale applications, they usually fail to offer satisfactory performance. As a huge amount of fine-grained knowledge is required to solve the ambiguity problem, it is quite difficult for rule-based approach to acquire the huge and fine-grained knowledge, and maintain consistency among them by human [Su 90a].

Probabilistic approaches attack these problems by providing a more objective measure on the preference to a given interpretation. Then, these approaches acquire huge and fine grained knowledge, or parameters in statistic terms from the corpus automatically. The uncertainty problem in linguistic phenomena is resolved on a more solid basis if a probabilistic approach is adopted. Moreover, the knowledge acquired by the statistical method is always consistent because the knowledge is acquired by jointly considering all the data in the corpus at the same time. Hence, the time for knowledge acquisition and the cost to maintain consistency are significantly reduced by adopting those probabilistic approaches.

To resolve the problems resulting from syntactic ambiguities, a unified statistical approach for ambiguity resolution has been proposed by $\mathrm{Su}[\mathrm{Su} 88$, 92b]. In that approach, all knowledge sources, including lexical, syntactic and semantic knowledge, are encoded by a unified probabilistic score function with a uniform formulation. This uniform probabilistic score function has been successfully applied in spoken language processing [Su 90b, 91b, 92a] and machine translation systems [Chen 91] to integrate different knowledge sources for ambiguity resolution.

In implementing this unified probabilistic score function, values of score functions are estimated from the data in the training corpus. However, due to the problem of insufficiency of training data and incompleteness of model knowledge, the statistical variations between the training corpus and the real application are usually not covered by this approach. Therefore, the performance in the testing set sometimes gets poor in the real application.

To enhance the capability of discrimination and robustness of those proposed score function, a discrimination-oriented adaptive leaming is proposed in this paper. And then, the robustness of this proposed adaptive leaming procedure is enhanced by enlarging the margin between the correct candidate and its confusing candidates to achieve maximum separation between different candidates.

Since the implementation of this adaptive learning procedure is based on the uniform probabilistic score function, we will first briefly review the unified probabilistic score function. Readers who are 
interested in the details about the uniform proba bilistic score function please refer [Chen 91, Su 91b, 92a, 92b].

\section{Overview of Uniform Probabilistic Score Function}

\subsection{General Definition}

A Score Function for a given syntactic tree, say $S y^{n}{ }_{j}$, is defined as follows:

$$
\text { Score }\left(S y n_{j}\right) \equiv \mu\left(S_{y n_{j}}, L_{e x} \mid w_{1}^{n}\right),
$$

where $w_{1}^{n}$ is the input word sequence, $w_{1}^{\text {th }}=$ $\left\{w_{1}, w_{2}, \cdots, w_{n}\right\}$, and $L e x_{j}$, the corresponding lexical string, i.e., part of speech sequence $\left\{c_{j_{1}}, c_{j_{2}}, \cdots, c_{j_{n}}\right\}$. By applying the multiplication theorem of probability, $I^{2}\left(S_{y} n_{1}, L e x, \mid w_{1}^{n}\right)$ can be rcstated as follows.

$$
\begin{aligned}
& P\left(S_{y n}, L, e x, \mid w_{1}^{*}\right) \\
& =P\left(S y n, \mid L e x_{3}, w_{1}^{\prime \prime}\right) \times P\left(L e x, \mid w_{1}^{\prime \prime}\right) \\
& =S_{\text {syn }}\left(S y n_{j}\right) \times S_{l e x}\left(L e x_{j}\right) .
\end{aligned}
$$

The two components, $S_{s y n}\left(S y n_{1}\right)$ and $S_{\text {tex }}\left(L_{e x}\right)$, in the above formula are called syntactic Score Function and Lexical Score Function, respectively. The original score function, i.e., $P\left(S y n_{1}, L e x, \mid w_{1}^{21}\right)$, is then called Integrated Score Function.

Next, we assume the information, from the word sequence $w_{1}^{n}$, required for syntactic ambiguity resolution, has percolated to the lexical interpretation $L e x_{j}$. Also, only little additional information can be provided from $w_{1}^{n}$ for the task of disambiguating syntactic interpretation $S y n_{j}$ after the lexical interpretation $l e x_{j}$ is given. Thus, the syntactic score can be approximated as shown in Eq.(3):

$$
S_{s y n}\left(S y n_{j}\right)=P\left(S y n, \mid L e x_{,}, w_{1}^{n}\right) \approx P\left(S y n_{j} \mid L e x_{\jmath}\right) .
$$

The integrated score function $P\left(S y n_{j}, L e x_{l} \mid w_{1}^{\mathrm{n}}\right)$ is then approximated as follows.

$$
\begin{aligned}
& P\left(S y n_{3}, L e x_{j} \mid w_{1}^{\prime \prime}\right) \\
& \approx P\left(S y n_{j} \mid L e x_{j}\right) \times P\left(L_{e x} \mid w_{1}^{n}\right) .
\end{aligned}
$$

Such a formulation allows us to use both lexical and syntactic knowledge in assigning preference measure to a syntactic tree. In the real computation, $\log$ operation is used to convent the operations of multiplication to the operations of addition. The following cquation shows the final form in the real application.

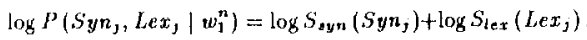

\subsection{Lexical Score Function}

Let $c_{k_{1}^{\prime \prime}}$ denote the $k-t h$ sequence of the lexical category, or part of speech, corresponding to the word scquence $w_{1}^{n}$. The Lexical Score Function can be expressed as follows [Chen 91, Su 92b]:

$$
\begin{aligned}
S_{\text {lex }}(\text { Lexk }) & =P\left(l \text { ex } \mid w_{1}^{n}\right)=P\left(c_{k_{i}^{n}} \mid w_{1}^{n}\right) \\
& =\prod_{i=1}^{n} P\left(c_{k,} \mid c_{k_{1}^{\prime-1}, w_{1}^{n}}^{n}\right),
\end{aligned}
$$

where $c_{k_{i}}$ is the lexical category of $w_{i}$. Several forms [Gars 87, Chur 88, Su 92b] for $P\left(c_{k} \mid c_{k^{\prime}-1}, w_{1}^{\prime \prime}\right)$ were proposed to simplify the computation. For examplc, [Chur 88] approximated $P\left(c_{k_{1}} \mid c_{k_{1}^{\prime-1}}, w_{1}^{n}\right)$ by $\left[P\left(c_{k_{1}} \mid c_{k_{1},-1}\right) \times P\left(c_{k_{1}} \mid w_{1}\right)\right] . A$ general nonlinear smoothing form [Chen 91] described in $\mathrm{Eq} .(7)$ is adopted in this paper:

$$
\begin{aligned}
& g\left(P\left(c_{k_{1}} \mid c_{k_{1}-1}, w_{i}\right)\right) \\
& \approx \lambda g\left(P\left(c_{k_{1}} \mid w_{i}\right)\right)+(1-\lambda) g\left(c_{k_{1}} \mid c_{k_{1-1}}\right),
\end{aligned}
$$

where $\lambda$ is the lexical weight $(\lambda=0.6$ is used in the current setup), and $g$ is a transform function $(\log (\cdot)$ is used in this paper). Hence, given both Eq.(6) and (7), the following formula is derived:

$$
\begin{aligned}
& \log \left(S_{\text {lex }}\left(\operatorname{Lex} x_{k}\right)\right) \\
& =\sum_{i=1}^{n}\left\{\lambda \log P\left(c_{k} \mid w_{i}\right)+(1-\lambda) \log P\left(c k_{1} \mid c_{k_{1-1}}\right)\right\} .
\end{aligned}
$$

It is noted that the above generalized form reduced to the formulation of [Chur 88] when the transfonm function is $\log$ function and $\lambda$ is 0.5 .

\subsection{Syntactic Score Function}

To show the computing mechanism for the syntactic score, we take the syntax tree in Fig. 1 as an example. The syntax tree is decomposed into a number of phrase levels. Each phrase level (also called a sentential form) consists of a set of synbols (terminal or nontenininal) which can derive all the teminal symbols in a sentence. Let label $t_{i}$ in Fig. 1 be the time index for cach state transition of a LR parser, and $L_{j}$ be the $i$-th phrase level. Thus, a transition from phrase level $L_{i}$ to phrase level $L_{i+1}$ is equivalent to a redue action at time $t_{i}$.

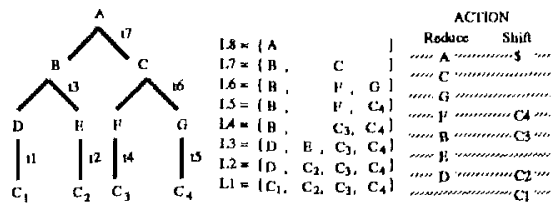

Figure 1 'The decomposition of a syntax tree into phrase levels. 
The syntactic score of the syntax tree in Fig.1 is then defined as

$$
\begin{aligned}
S_{\text {syn }}\left(\operatorname{syn}_{A}\right) & \equiv P\left(L_{8}, L_{7}, \cdots, L_{2} \mid L_{1}\right) \\
& =\prod_{i=2}^{8} P\left(L_{i} \mid L_{1}^{i-1}\right) \approx \prod_{i=2}^{8} P\left(L_{i} \mid L_{i-1}\right),
\end{aligned}
$$

where $\operatorname{syn} n_{A}$ is the parse trec, and $\mathrm{L}_{1}$ through $\mathrm{L}_{8}$ represent different phrase levels. Note that the product terms in the last formula correspond to the rightmost derivation sequence in a general LR parser [ $\mathrm{Su}$ 91c], with left and right contexts taken into account. Therefore, such a formulation is especially useful for a generalized LR parsing algorithm, in which context-sensitive processing power is desirable.

Although the context-sensitive model in the above equation provides the ability to deal with intra-level context-sensitivity, it fails to catch interlevel correlation. In addition, the formulation of Eq.(9) gives rise to the normalization problem for ambiguous syntax trees with different number of nodes. An altemative to relieve this problem is to compact multiple highly correlated phrase levels into one in evaluating the syntactic scores. The formulation is expressed as follows [Su 91c]:

$$
\begin{aligned}
& S_{s y n}\left(\operatorname{syn}_{1}\right) \\
& \approx P\left(\mathrm{~L}_{k}, \mathrm{~L}_{i}, \mathrm{~L}_{43} \mid \mathrm{L}_{k}\right) \times P\left(\mathrm{~L}_{4} \mid \mathrm{L}_{4}\right) \times P\left(\mathrm{~L}_{4}, \mathrm{~L}_{3} \mid \mathrm{L}_{2}\right) \times P\left(\mathrm{~L}_{i} \mid \mathrm{L}\right. \\
& \approx P\left(\mathrm{~L}_{*} \mid \mathrm{L}_{5}\right) \times P\left(\mathrm{~L}_{4} \mid \mathrm{L}_{4}\right) \times P\left(\mathrm{~L}_{4} \mid \mathrm{L}_{2}\right) \times P\left(\mathrm{~L}_{2} \mid \mathrm{L}_{1}\right)
\end{aligned}
$$

Because the number of shifts, i.c., the number of terms in Eq.(10), is always the same for all ambiguous syntax trees, the normalization problem is then resolved. Moreover, it provides a way to consider both intra-level context-sensitivity and inter-level correlation of the underlying context-free grammar. With such a score function, the capability of context-sensitive parsing (in probability sense) can be achieved with a context-free grammar.

\section{Discrimination and Robustness Oriented Adaptive Learning}

\subsection{Concepts of Adaptive Learning}

The general idea of adaptive leaming is to adjust the model parameters (in this paper, they are lexical scores and syntactic scores) to achieve the desired criterion (in our case, it is to minimize the error rate). To explain clearly how the adaptive leaming works, we take the sentence "I saw a man." as an example. The lexical category (i.e., part of speech) and its corresponding log score for each word are listed in Table 1.

\begin{tabular}{|c|c|c|}
\hline Word & $\begin{array}{c}\text { Category } \\
\text { (part of specch) }\end{array}$ & $\log \mathbf{P}(\mathbf{c} \mid \mathbf{w})$ \\
\hline \multirow{2}{*}{$\mathrm{N}$} & pron (pronoun) & -0.22 \\
& $\mathrm{n}$ (noun) & -0.39 \\
\hline \multirow{2}{*}{ saw } & vi (intransitive verb) & -0.52 \\
& vt (transitive verb) & -0.16 \\
\hline \multirow{2}{*}{$a$} & art (article) & -0.02 \\
& prep (preposition) & -1.30 \\
\hline man & $\mathbb{n}$ (noun) & 0 \\
\hline
\end{tabular}

Table 1 Categories for words and thelr log word-to-category scores.

In Table 1 , the log word-to-category scone, $\log (P(c \mid w))$, for each word is estimated from the training corpus by calculating their relative frequencies. For example, in the training corpus, the word " $l$ " is used as pronoun for 60 times, and 40 times as noun. Then, the log word-to-category scores can be calculated as follows.

$$
\begin{gathered}
\log _{10} P\left(\text { pron } \mid\{[\})=\log _{10}\left(\frac{60}{60+40}\right)=-0.22,\right. \\
\log _{10} P(\mathrm{n} \mid\{\mathrm{I}\})=\log _{10}\left(\frac{40}{60+40}\right)=-0.39 .
\end{gathered}
$$

In this example, there are $2 * 2 * 2 * 1=8$ possible different ways to assign lexical categories to the input sentence. When these 8 possible lexical sequences are parsed, only four of them are accepted by our parser. They are listed as follows:

1. pron $\mathrm{vt}$ art $\mathrm{n}$

2. n vt art $n$

3. pron vi prep $\mathrm{n}$

4. n vi prep $n$.

The syntactic scores of different parse trees are then calculated according to Eq.(10). A parse trec corresponding to the lexical sequence "Ipron vt art n]" is drawn below as an example.

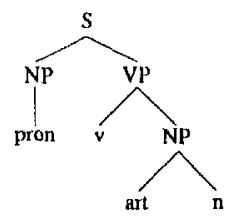

The log syntactic scores for those four grammatical inputs are computed and listed in Table 2. 


\begin{tabular}{|c|c|}
\hline Input Lexical Sequence & log syntuctic score \\
\hline [pron vt art n] & $(-0.7)+(-0.3)+(-0.3)+(-0.2)=-1.5$ \\
\hline [n vt ant n] & $(-0.2)+(-0.3)+(-0.3)+(-0.2)=-1.0$ \\
\hline [pron vi prep n] & $(-0.7)+(-0.7)+(-0.4)+(-0.3)=-2]$. \\
\hline [n vi prep n] & $(-0.2)+(-0.7)+(-0.4)+(-0.3)=-1.6$ \\
\hline
\end{tabular}

Table 2 log syntactic scores of the grammatical input lexical sequences.

According to Eq.(5), the total log integrated score $\left(\log S_{l e x}+\log S_{s y n}\right)$ for each parsed sentence hypothesis is calculated. For example, the log lexical score for "l/[pron] saw/[vt] a/[art] man/[n]" = ($0.22-0.16-0.02-0)=-0.4$. Finally, the $\log$ integrated scores for the above grammatical inputs are listed as follows:

candidate $-1 . \quad \log$ integrated score $=(-0.40-1.5=-1.90)$ : I/[pron] saw/[vt] a/[art] man/[n]

candidate $-2 . \quad \log$ integrated score $=(-0.57-1.0=-1.57): \mathrm{V} /[\mathrm{n}]$ saw/[vt] a/[art] man/[n]

candidale $-3 . \quad \log$ integrated score $=(-2.04-2.1=-4.71)$ : I/[pron] saw/[vi] a/[prep] man/[n]

candidate $-4 . \quad \log$ integrated score $=(-2.21-1.6=-3.81): \mathrm{J} /[\mathrm{n}]$ saw/[vi] a/[prep] $\operatorname{man} /[n]$

Among these four candidates, the candidate 1 is regarded as the desired selection by linguists. Since our decision criterion will select the candidate which has the highest integrated score, i.e., the second one; $\mathrm{I} /[\mathrm{n}] \mathrm{saw} / \mathrm{vt}] \mathrm{a} /[\mathrm{art}] \mathrm{man} /[\mathrm{n}]$, it results in a decision error in this case.

To remedy this error, adaptive leaming procedure is adopted to adjust the score values iteratively, including lexical and syntactic scores, until the integrated score of the correct candidate (i.e., candidate 1) raises to the highest rank. In this paper, parameters which are adjusted by adaptive learning procedure are those $\log$ scores, including $\log P\left(c_{k_{i}} \mid w_{i}\right)$, $\log P\left(c_{k_{i}} \mid c_{k_{1}^{i-1}}\right)$ and $\log P\left(\mathrm{~L}_{\mathrm{i}} \mid \mathrm{L}_{1}^{\mathrm{i}-1}\right)$. The amount of adjustment in each iteration depends on the misclassification distance. Misclassification distance is defined as the difference between the score of the top candidate and that of the correct one. (In the above example, distance $=$ (score of correct candidate)-(score of top candidate) $=(-1.90)$ $(-1.57)=-0.33)$. From iteration to iteration, the parameters (both lexical and syntactic scores) are adjusted so that the integrated score of the correct candidate is increased, and the integrated score of the wrong candidate is decreased at the same time. The learning procedure for a sentence is stopped when the candidate of this sentence is correctly selected. To make the explanation of this adaptive learning procedure clear, we assume lexical scores are unchanged during leaming. That is, only the parameters of the syntactic scores are adjusted. The details of adaptive learning for adjusting syntactic scores are listed as follows:

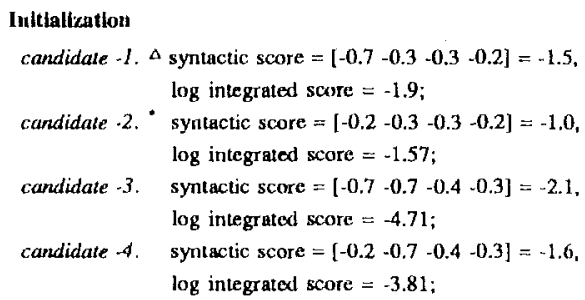

\section{Iteration 1}

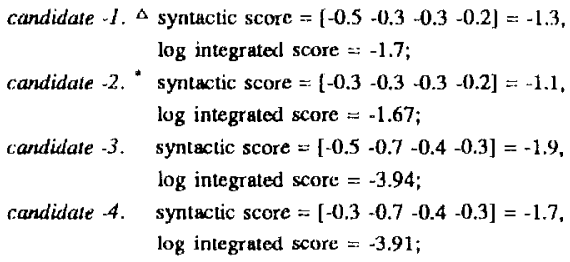

\section{Iteration 2}

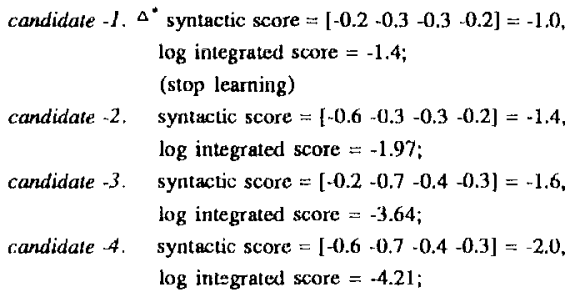

(where* denotes the top candidate, und $\Delta$ demotes the desired candidate)

It is clear that after the second iteration, parameters have been adjusted so that the desired candidate (i.e., candidate 1) would be selected.

\subsection{Procedure of Discrimination Learning}

Since correct decision only depends upon correct rank ordering of the integrated scores for all ambiguitics, not their real value, a discriminationoriented approach should directly pursue correct rank ordering. To derive the discrimination function, the probability scores mentioned above are first jointly considered. Then, a discrimination-oriented function, namely $g(\cdot)$, is defined as a measurement of above mentioned score functions, so that it can well preserve the correct rank ordering [Su 91a]. Here, $g(\cdot)$ is chosen as the weighted sum of log 
lexical and log syntactic scores, i.e.,

$$
\begin{aligned}
& g\left(S y n_{k}\right) \\
& =w_{l e x} \cdot \log S_{l e x}\left(L e x_{k}\right)+w_{\Delta y n} \cdot \log S_{s y n}\left(S y n_{k}\right) \\
& =w_{l e x} \cdot \sum_{i=1}^{n} \log P\left(c_{k_{i}} \mid c_{k_{i}+-1}, w_{1}^{n}\right)+w_{s y n} \cdot \sum_{i=1}^{n} \log P\left(L_{i} \mid L_{1}^{i-1}\right) \\
& =w_{\text {lex }} \cdot \sum_{i=1}^{n} \lambda_{\text {lex }}(i)+w_{s y n} \cdot \sum_{i=1}^{n} \lambda_{s y n}(i)
\end{aligned}
$$

where $\lambda_{\text {lex }}(i)=\log P\left(c_{k_{i}} \mid c_{k_{j}^{\prime}-1}, w_{i}^{n}\right)$, and $\lambda_{s y n}(i)=$ $\log P\left(L_{i} \mid L_{1}^{i-1}\right)$. Both stand for the $\log$ lexical score and the log syntactic score of the $i$-th word for the $k$-th syntactic ambiguity, respectively. In addition, $w_{l e x}$ and $w_{s y n}$ correspond to the wcights of lexical and syntactic scores, respectively.

If the parse tree of a sentence is misselected, the parameters (i.e., the lexical and the syntactic scores) are adjusted via the proposed adaptive learning procedure. Otherwise, no parameters would be adjusted. When misselection occurs, the misclassification distance, $d_{s c}$, is less than zero. This misclassification distance is defined as the difference between the log integrated score of the correct candidate and that of the top one. A specific term of the syntactic score components in the $(t+l)$-th iteration of the correct candidate, say $\lambda_{s y n}^{(t+1)}(j)$, would be adjusted as follows:

$$
\begin{cases}\lambda_{s y n}^{(t+1)}(j)=\lambda_{s y n}^{(t)}(j)+\Delta \lambda_{s y n}^{(t)}(j), & d_{s c} \leq 0, \\ \lambda_{s y n}^{(t+1)}(j)=\lambda_{s y n}^{(t)}(j), & \text { otherwise. }\end{cases}
$$

At the same time, the term of the syntactic score components of the top candidate would be adjusted according to the following formulas:

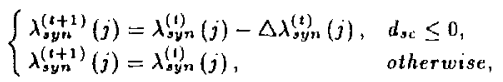

where $\Delta \lambda_{s y n}^{(t)}(j)$ is the amount of adjustment. This value is represented as

$$
\Delta \lambda_{a y n}^{(t)}(j)=\frac{\epsilon \cdot d_{0}}{d_{a c}^{2}+d_{0}^{2}} \cdot \frac{w_{a y n}}{\sum_{i=1}^{n}\left[w_{l e x} \cdot \lambda_{l e s}^{(t)}(i)+w_{\text {ayn }} \cdot \lambda_{a y n}^{(t)}(i)\right]},
$$

where $d_{0}$ is a constant which stands for a window size, and $\epsilon$ is the learning constant for controlling the speed of convergence. The leaming rule for adjusting the lexical scores can be represented in a similar manner. Notice that only the parameters of the top candidate and those of the correct candidate would be adjusted when misselections occur. Those parameters of other wrong candidates would not be adjusted in this adaptive learning procedure. From Eq.(13), (14) and (15), it is clear that the score of the correct candidate will increase and that of wrong candidate will decrease from iteration to iteration until the correct candidate is selected. For the purpose of clarity, the detailed derivations of the above adaptive learning procedure will not be given here. Interested readers can contact the authors for details.

\subsection{Robustness Issues}

Since it is easy to improve the performance in a training set by adopting a model with a large number of parameters, the error rate measured in the training sct frequently tums out to be over-optimistic. Moreover, the parameters estimated from the training corpus may be quite differ from that obtained from the real applications. These phenomena may occur due to the factors of finite sampling size, style mismatch, or domain mismatch, etc. To achieve a better perfonmance in the real application, one must deal with the possible mismatch of parameters, or statistical variation between the training corpus and the real application. One way to achieve this goal is to enlarge the inter-class distance to achieve maximum separation [Su 91a] between the correct candidate and the other candidates. That is, this approach provides a tolerance zone between different candidates for allowing possible data scattering in the real application.

Traditional adaptive leaming methods [Amar 67. Kata 90] stop adjusting parameters once the in put pattern has been correctly classified. However, if we stop adjusting parameters under the condition that the observations are correctly classified in the training corpus, the distance between the correct candidatc and other ambiguities may still be too small. Thus, it is vulnerable to deal with possible modeling errors and statistical variations between the training corpus and the real application. Su [Su 91a] has proposed a robust learning procedure which continues to enlarge the margin between the correct candidate and the top one, even if the syntax tree of the sentence has been correctly selected. That is, the parameters will not be adjusted only if the distance between the correct candidate and the others has exceeded a given threshold. The leaming rules in Eq.(13), (14) are then modified as follows.

If $d_{s c} \leq \delta$, where $\delta$ is a preset margin, the syntactic score in the $(t+1)$ iteration for the correct candidate is adjusted according to the following formulas:

$$
\begin{cases}\lambda_{s y_{n}}^{(i+1)}(j)=\lambda_{s y n}^{(t)}(j)+\Delta \lambda_{s y n}^{(t)}(j), & d_{s c} \leq \delta, \\ \lambda_{s y n}^{(t+1)}(j)=\lambda_{s, n}^{(t)}(j), & \text { otherwise. }\end{cases}
$$

And, the syntactic score of the top candidate is adjusted as follows:

$$
\begin{cases}\lambda_{s, n}^{(t+1)}(j)=\lambda_{s y n}^{(t)}(j)-\Delta \lambda_{s y n}^{(t)}(j), & d_{o c} \leq \delta . \\ \lambda_{b y+1}^{(+1)}(j)=\lambda_{s y n}^{(!)}(j), & \text { otherwise. }\end{cases}
$$




\section{Simulations}

The following expcriments are conducted to investigate the advantage of the proposed discrimination and robustness oriented adaptive leaning procedure. In the experiments, 4,000 sentences, which are extracted from IBM techuical manuals are first associated with their corresponding correct category sequences and correct parsed trees by linguists. The corpus are then partitioned into a training corpus of 3,200 sentences and a test set of 800 sentences. Next, the lexical and syntactic probatilities are estimated from the data in the training conpus. Afterwards, the sentences in the test set are used to evaluate the performance of the proposed algorithm using the estimated lexical and syntactic probabilities. This integrated score function approach using the estimated probabilitics is considered as the baseline system. Performances of discrimination oriented adaptive learnings with and without robusiness enhancement are then cvaluated. The accuracy rate of the syntactic ambiguity resolution for the training corpus and the test set are summarized in Table 3. (Note that the top candidate is selected from all possible parses allowed by the grammars of the system; therefore, the bascline perfonnance is evaluated under a highly ambiguous cuvionment.)

\begin{tabular}{|c|c|c|}
\hline Baseline & $\begin{array}{c}\text { Training } \\
\text { Corpus }\end{array}$ & Test Set \\
\hline $\begin{array}{c}\text { + Basic version of } \\
\text { learning }\end{array}$ & 79.75 & 46.00 \\
\hline $\begin{array}{c}\text { Robust version of } \\
\text { Learning }\end{array}$ & 95.50 & 56.88 \\
\hline
\end{tabular}

Table 3 Aecuracy rate (in \%) of syntactic disamblguation

Table 3 shows that syntax trec accuracy ratc is improved from $46 \%$ to $56.88 \%$ using the basic version of discrimination oricnted adaptive leaning procedure. This significant improvement shows the superiority of the adaptive lcarning procedure for dealing with the disambiguation lask. Funbennore, when the robust version of Icaming procedure is adopted, the performance is improved further (from $56.88 \%$ to $60.62 \%$ ). It means that the robustness of the learning procedure is indeed cnlanced by $\mathrm{cm}-$ larging the distance between the correct candidate and other candidates. Morcover, not only the accuracy rate of syntax tree is improved using adaptive leaming, but also that of lexical sequence is improved. In this paper, a lexical sequence is $1 \mathrm{c}-$ garded as "correct" only if all the lexical categories in a sentence perfectly match those selected by linguists. In other words, we are measuring "sentence accuracy rate" in contratet to "word accuracy rate" as adopted in [Chur 88, Giars 87]. Table 4 shows that the basic version of adaptive learning procedure improves the sentence accuracy rate of lexical sequences absut $5 \%$ (froin $77.12 \%$ to $82.38 \%$ ). Again, with the robust version of leaming, the accurate rate of lexical sequences is greatly enhanced.

\begin{tabular}{|c|c|c|}
\hline Huseline & $\begin{array}{c}\text { Truinlng } \\
\text { Corpus }\end{array}$ & Test Set \\
\hline+ Hasic version of learning & 91.41 & 77.12 \\
\hline $\begin{array}{c}\text { Kobust verslon of } \\
\text { Learning }\end{array}$ & 98.91 & 82.38 \\
\hline
\end{tabular}

Table 4 Sentence uecuracy rate (in \%) of lexical sequences

The behavior of each iteration of the adaptive leaming process is shown in Figure 2. Through ob scrving this figure, we can conclude that if the robustness issues are not considered during leaming, the performance of the test set would decrease as the training process goes on. This is the phenomena of over-tuning. However, by forcing the learning procedure to continue until the separation between the correct candidate and the top one exceeds the desired margin, the perfomance of the test set can be further improved, and no degradation phenomenon is observed.

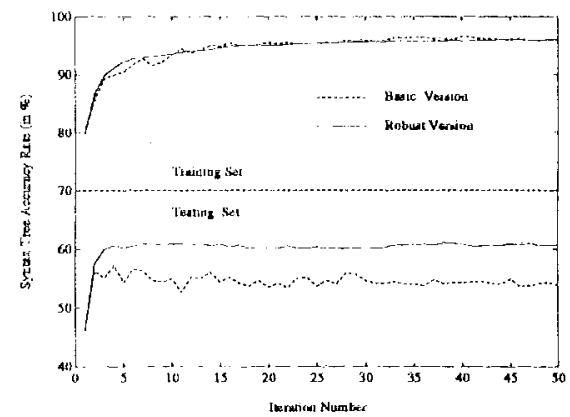

ligure 2 Syntax tree accuracy rate versus iterations for busic and robust version of adaptive learning 


\section{Summary}

Because of insufficient training data, and approximation error introduced by the language model, traditional statistical approaches, which resolve ambiguities by indirectly and implicitly using maximum likelihood method, fail to achieve high performance in real applications. To overcome these problems, adaptive learning is proposed to pursue the goal of minimizing discrimination error directly. The performance of syntactic ambiguity resolution is significantly improved using the discrimination oriented analysis. In addition, the sentence accuracy rate of the lexical sequences is also improved. Moreover, the performance is further enhanced by using the robust version of learning procedure, which enlargeds the margin between the correct candidate and its candidates. The final results show that using the basic version of leaming, the syntax tree selection accuracy rate is improved about $10 \%$ (from $46 \%$ to $56.88 \%$ ), and the total improvement is over $14 \%$ using robust version leaming. Also, the sentence accuracy rate for lexical sequences is improved from $77.12 \%$ to 82.38 and $87.88 \%$ using the basic and robust version of leaming procedure, respectively.

\section{Reference}

[Amar 67] Amari S., "A theory of adaptive pattern classifiers," IEEE Trans. on Electronic Computers, Vol. EC-16, pp. 299-307, June 1967

[Chen 91] Chen S.-C., J.-S. Chang, J.-N. Wang, and K.-Y. Su, "ArchTran: A Corpusbased Statistics-oriented English-Chinese Machine Translation System," Proc. of Machine Translation Summil III, Washington, D. C., U.S.A., July 1-3, 1991.

[Chur 88] Church, K., "A Stochastic Parts Program and Noun Phrase for Unrestricted Text," ACL Proceedings of 2nd Conference on Applied Natural Language Processing, pp.136-143, Austin, Texas, U.S.A., 9-12 Feb. 1988.

[Gars 87] Garside, R., G., Lech, and G., Sampson, "The Computational Analysis of English: A Corpus-Based Approach," London: Longman.

[Kata 90] S.Katagiri. C.H. Lee, "A Generalized Probabilistic Decent Method," Proc.
Acous. Sco. of Japan, 2-p-6, pp. 141-142, Nagoya, Sept 1990.

[Su 88] Su K.-Y., and J.-S. Chang, "Semantic and Syntactic Aspects of Score Function," Proc. COLING-88, Vol.2, pp. 642-644, 12th Int. Conf. on Comput. Linguistic, Budapest, Hurgay, 22-27, Aug. 1988.

[Su 90a] Su, K.-Y., and J.-S Chang, 1990. "Some Key Issues in Designing MT Systems," Machine Translation, vol. 5, no. 4, pp. 265-300, 1990.

[Su 90b] Su K.-Y., T.-H. Chiang and Y.-C. Lin, "A Unified Probabilistic Score Function for Integrating Speech and Language Information in Spoken Language Processing," Proceeding of 1990 Intemational Conference on Spoken Language Processing, pp.901-904, Kobe, Japan, 19-22 Nov. 1990.

[Su 91b] Keh-Yih Su, Tung-Hui Chiang and YiChung Lin, "A Robustness and Discrimination Oriented Score Function for Integrating Speech and Language Processing," Proceeding of the 2nd European Conference on Speech Communication and Technology, Genova, Italy, pp. 207-210, Sep. 24-261991.

[Su 91a] Su K.-Y., and C.-H. Lee, "Robustness and Discrimination Oriented Speech Recognition Using Weighted HMM and Subspace Projection Approaches," Proc. ICASSP91, pp.541-544, Toranto, Canada, 14-17 May, 1991.

[Su 9lc) Su K.-Y., J.-N. Wang, M.-H. Su, and J.S. Chang, "GLR Parsing with Scoring," in Tomita, Masaru (ed.), Generalized LR Parsing, Chapter 7, pp.93-112, Kluwer Academic Publisher 1991.

[Su 92a] Keh-Yih Su, Tung-Hui Chiang and YiChung Lin, "An Unified Framework to Incorporate Speech and Language Information in Spoken Language Processing" to appear in the Procceding of IEEE International Conference on Acoustics, Speech and Signal Processing, ICASSP-92, San Francisco, Califomia, U.S.A., March 23261992.

[Su 92b] Su K.-Y., J.-S. Chang and Y.-C. Lin, "A Unified Approach to Disambiguation Using A Uniform Formulation of Probabilistic Score Function," in preparation. 